\section{E-055 FULLY ENDOSCOPIC CLIPPING OF ANTERIOR CIRCULATION ANEURYSMS: A SURGICAL STEP CLOSER TO ENDOVASCULAR BENEFITS?}

${ }^{1} \mathrm{~S}$ Koester*, ${ }^{2} \mathrm{R}$ Bertani, ${ }^{3} \mathrm{C}$ Eduardo Ferrarez. ${ }^{1}$ Neurologic Surgery, Vanderbilt University Medical Center, Nashville, $T N_{;}{ }^{2}$ Hospital Municipal Miguel Couto, Rio de Janeiro, Brazil; ${ }^{3}$ Neurologic Surgery, Hospital Municipal Miguel Couto, Rio de Janeiro, Brazil

10.1136/neurintsurg-2021-SNIS.150

Introduction Advancement of endovascular treatment modalities with pipeline flow diversion, stenting, and coiling for aneurysms has pushed against the frontier set by open surgical clipping and bypass. Endovascular treatment has historically shown a lower risk profile, lower readmission rates with certain procedures, and a more favorable cosmetic result, pushing centers to perform a greater proportion of procedures endovascularly. With our cases of fully endoscopic clipping of anterior circulation aneurysms through minimally invasive keyhole craniotomies, we exhibit open neurosurgery's potential transition to "new" standards for absence of cosmetic defects, a less invasive approach, and less exposure to radiation.

Case Presentations A 67-year-old female presented with persistent headaches, and was found to have an unruptured, right MCA bifurcation aneurysm upon CTA examination. The next, a 45 -year-old patient, also with persistent headaches, was found to have an unruptured ICA aneurysm. Both lack significant comorbidities and report being previously healthy. Fully endoscopic treatment through a transpalpebral incision was elected to be used to treat both unruptured aneurysms.

Results No intra-operative complications were experienced, and the surgical field remained well-visualized throughout the procedure with the endoscope. Patients remained neurologically and hemodynamically stable during and after surgery, there were no post-operative complications. Postoperative imaging showed adequate clipping of the aneurysms. The patients both had minimal cosmetic defects.

Conclusion The use of a fully endoscopic approach shows potential for improving the quality of care through reduced operative time, lower complications, and improved craniofacial aesthetic to the patient. We believe that fully endoscopic clipping of select non-ruptured aneurysms is a safe and effective alternative surgical option that can be performed through minimally invasive approaches with a lower risk profile and favorable cosmetic result, potentially comparable to that of endovascular treatment.

Disclosures S. Koester: None. R. Bertani: None. C. Eduardo Ferrarez: None.

\section{E-056 ANATOMICAL FINDINGS AT THE CIRCLE OF WILLIS AND CERVICAL CAROTID ARTERIES DURING LARGE VESSEL OCCLUSION STROKE: OBSERVATIONS FROM COMPUTED TOMOGRAPHY ANGIOGRAPHY}

R Kiwan*, S Lownie. Western University, London, ON, Canada

\subsection{6/neurintsurg-2021-SNIS.151}

Objective The circle of Willis (CoW) and cervical carotid arteries are potentially important sources of collateral flow (or flow limitation) during acute large vessel occlusion (LVO) in the anterior circulation. We sought to examine the anatomical components of the circle and the cervical carotid arteries to determine whether they bear any relationship to acute stroke severity.

Methods Consecutive patients with acute LVO who underwent endovascular thrombectomy (EVT) between September 2018 and June 2019 were assessed using computed tomography angiography (CTA). Measurements were made of the luminal diameters of 16 anatomical vascular components of the CoW and neck. Measures of stroke severity, including the admission NIHSS, ASPECTS and mCTA collateral scores, were statistically analyzed for any relationship to vascular measurements.

Results One hundred patients (51 men, 49 women) between 32 and 99 years of age (mean 72.3 years) were studied. No relationship was found between the anterior or posterior collateral Willis pathways and measures of stroke severity. On the other hand, the ophthalmic arteries did exhibit a relationship to stroke severity. In adjusted analysis, a 1-mm increase in the ipsilateral and contralateral ophthalmic artery diameter was independently associated with a 4.80 -point decrease $(95 \%$ CI: $1.26,8.34)$ and a 6.31-point increase (95\% CI: 0.27 , 12.36) in the NIHSS scale, respectively. Similarly, 1-mm increases in the ipsilateral and contralateral ophthalmic artery diameter were respectively associated with a 1.53-point increase $(95 \%$ CI: $0.66,2.41)$ and a 2.62 -point decrease $(95 \%$ CI $1.12,4.13)$ in the ASPECTS. In the neck, ipsilateral carotid artery analysis showed a majority with $0 \%$ stenosis, and unexpectedly no NASCET stenosis between 55\% and 95\%, while a minority (14\%) were $95 \%$ to $100 \%$.

Conclusions Stroke severity and native collateral during LVO is unrelated to circle of Willis anatomy. Ophthalmic artery calibers show some relationship to stroke severity. Our findings also support the notion of acute progression of moderate and severe NASCET stenoses to near occlusion or complete occlusion at the onset of LVO stroke, such that no cases exhibited moderate or severe $55-95 \%$ narrowing.

Disclosures R. Kiwan: None. S. Lownie: None.

\section{E-057 PROSPECTIVE PREHOSPITAL EVALUATION OF VAN AND RACE LARGE VESSEL OCCLUSION PREDICTION INSTRUMENTS ON A MOBILE STROKE UNIT}

J Hosny*, J Lynch, A De Havenon, M Tanweer, M Alahmad, O Zaidat. Bon Secours Mercy Health St Vincent Medical Center, Toledo, $\mathrm{OH}$

10.1136/neurintsurg-2021-SNIS. 152

Background and Purpose A variety of large vessel occlusion (LVO) prediction instruments have recently been developed with the intention of appropriately routing patients to either the closest hospital, or to a hospital capable of performing endovascular thrombectomy. However, there is currently a scarcity of prospective data validating these instruments in the field. This study directly compares the efficacy of two instruments, the Rapid Arterial Occlusion Evaluation (RACE) scale, and the Vision, Aphasia, Neglect (VAN) screening tool.

Methods VAN and RACE were concurrently evaluated by the same observer on each patient transported by our mobile stroke unit (MSU). LVO status was determined by computed tomography angiography, magnetic resonance angiogram, or digital subtraction angiography within 24 hours. To compare the ability of VAN and RACE to detect LVO, we calculated their sensitivity, specificity, positive and 